\title{
Martín Heidegger: crítica al concepto hegeliano de experiencia (segunda parte)
}

\author{
Rodolfo García Aguilar \\ Tutor de la Cátedra de Filosofía. Departamento de Ciencias Sociales y Humanidades. Universidad Estatal a Distancia. \\ Correo electrónico:rga3cr@uned.ac.cr
}

\author{
Recibido: Febrero 2014 • Aceptado: Julio 2014
}

\begin{abstract}
RESUMEN
El presente trabajo tiene como objetivo retomar la temática expuesta por Martín Heidegger, en su artículo "El concepto hegeliano de experiencia", para rescatar sus reflexiones en el campo de la ontología. Se busca abrir nuevamente la discusión en un mundo metafísico donde se consolida lo que el autor llama "la imagen del mundo", y donde se considera esta, erróneamente, como la única forma de enfrentarse y apoderarse de la realidad.
\end{abstract}

Palabras clave: Heidegger, Hegel, experiencia, imagen del mundo, ser, apariencia, ente.

\section{ABSTRACT}

The objective of the present work is to take back the topic expounded by Martin Heidegger in his work Hegelian concept of experience, to rescue his reflections in the ontology field. To open again the discussion in a metaphysical world where de image of world consolidates it self, erroneously, as the only way to take possession of reality.

Key words: Heidegger, Hegel, experience, world image, being, appearance, entity.

\section{Introducción}

El presente es el segundo y último artículo de una serie de dos, en los cuales se comenta y analiza la exposición que hace Martín Heidegger (1960) en su obra El concepto hegeliano de experiencia, donde trata de evidenciar y demostrar que la posición hegeliana es una forma de metafísica, la cual consolida lo que él considera una imagen del mundo, nociva para la propia metafísica, puesto que cosifica y determina la fiscis como un dato con el cual cuenta la conciencia para consolidarse, como el punto de referencia de esta, es una tautología, un círculo que limita el conocimiento de la realidad.
La posición hegeliana se aleja de la verdadera realidad del ser, según Heidegger (1960). Ese ser debe buscarse nuevamente como parámetro de movimiento de la razón en un mundo regido por el cartesianismo. Debido a esto, ha ido perdiendo su vitalidad y ha quedado como una especie de vitrina, donde la conciencia se refleja y se justifica como el parámetro. Incluso, se podría decir que ha dejado su dinámica, su movimiento.

La metodología aplicada en esta exposición se basó en la selección de algunos de los capítulos del texto de Hegel, con el fin de seguir la exposición e interpretación hecha por Heidegger de ese autor. 
Heidegger va exponiendo el pensamiento de Hegel, usando como parámetro la obra Fenomenología del espíritu y, a partir de esta, desarrolla la concepción propia heideggeriana.

La exposición realizada de Hegel por parte de Heidegger es muy objetiva; sin embargo, el interés de este artículo es probar o verificar si la posición de Heidegger supera la posición clásica dialéctica de Hegel.

La exposición se iniciará a partir del capítulo octavo del artículo El concepto hegeliano de experiencia, puesto que en el primer artículo del autor del presente trabajo se agotaron los primeros siete capítulos. ${ }^{1}$

\section{Desarrollo}

\section{Capítulo octavo}

El capítulo octavo de la obra Fenomenología del espíritu, de Hegel, según la versión de Heidegger (1960), define el carácter del movimiento de la marcha histórica en la que transcurre la historia de la formación de la conciencia, donde el avance a través de la serie completa de las formas del saber tiene que resultar por sí mismo, en el sentido de la fuerza, de la energía del movimiento, de la dialéctica. Esta idea refleja el pensamiento de Hegel, y es lo manifiesto de todo el proceso interno que lleva la conciencia en su comportamiento dialéctico.

Recuérdese que el método dialéctico, el movimiento, en este caso de la conciencia, es la idea fundamental del pensamiento idealista, representado por Hegel.

Así las cosas, Hegel (en la traducción que del texto hace Heidegger, 1960) formula tres proposiciones sobre la conciencia. En el capítulo octavo, aparece la primera de ellas: "la conciencia se entera de su esencia de ser ciencia en el sentido de saber absoluto" (Hegel citado por Heidegger,

1. Los primeros siete capítulos de la obra de Guillermo Federico Hegel fueron comentados en un primer artículo, que fue publicado en el número 28 de esta misma Revista Espiga.
1960: 134). En esta proposición, se descubre un doble carácter de la conciencia: la conciencia se instituye en su aparecer, pero al mismo tiempo a la luz de su propia esencia. Se instituye en los aspectos esenciales de su aparecer y se organiza de esta suerte como reino de sus formas.

Para Hegel existen dos tipos de conciencia. Una limitada a su espacio y al entorno que crea, haciendo de ello el medio de su existencia; es su todo, su mundo; no existe otra forma de ser. Por otro lado, tenemos una conciencia crítica, real, que cuestiona el fundamento de esta; es el campo de la filosofía y de las ciencias; es salir de la inmediatez del mundo creado como protección a un mundo conceptual universal.

La conciencia no debe considerarse exclusivamente conciencia natural, ni exclusivamente conciencia real. Tampoco es la amalgama de ambas; por lo contrario, la conciencia misma es la unidad de ambas.

La conciencia misma es en sí la inquietud del distinguirse entre el saber natural y el saber real. "El movimiento de la marcha histórica se funda en esa inquietud de la conciencia misma y de ella tiene ya la dirección. La conciencia no se pone a posteriori en el movimiento ni recibe primero su dirección" (Hegel citado por Heidegger, 1960: 134). Por lo tanto, no son elementos inertes en la conciencia ni el saber real ni el saber natural; la conciencia es uno y otro.

Consecuentemente, entender el método dialéctico aplicado a la conciencia requiere utilizar e interpretar los conceptos de una forma diferente, y navegar en ellos con las condiciones que da el actor, en este caso, los actores.

Siguiendo con el desarrollo del tema, en este curso de la historia de la formación, la conciencia natural demuestra "ser solo concepto del saber", donde ese "solo" le basta a Hegel, debido a que la conciencia natural, en su representar lo existente, representa a la vez ineluctablemente la realidad de lo existente, aunque de modo no expreso. Heidegger (1960) afirma, a modo de crítica, que la conciencia natural está en su representar más allá de sí misma, aunque sin haber salido de sí.

Revista ESPIGA Año XIV, N. ${ }^{\circ}$ 29:45-53, enero-junio 2015 
Por otra parte, la versión clásica hegeliana considera que la conciencia natural tiene su propio opinar por verdadero, pero su propio opinar delata incesantemente la inquietud de ir más allá. La realidad de lo real es la meta, es decir, anticiparse la meta. Por eso, Hegel dice: "Pero la meta está encerrada en el saber tan necesariamente como la serie del avance" (Hegel citado por Heidegger, 1960: 135).

La idea anterior es expuesta por Heidegger (1960: 135) de la siguiente manera:

El avance en la marcha histórica de la historia de la formación de la conciencia, no es impulsado hacia adelante por la forma de la conciencia en cada momento, ni hacia lo aún indeterminado, sino que se infiere del fin ya puesto. La meta atrayente se pone de manifiesto ya en el atraer mismo en su aparecer y lleva de antemano la marcha de la conciencia hacia lo completo de su integridad.

En este sentido, la inquietud de la conciencia comienza constantemente el movimiento, lo que quiere decir que la skepsis dominante en la esencia del saber ha abarcado ya todas las posibles formas de la conciencia.

Este arrebatamiento de la conciencia del saber natural hacia el saber real implica la muerte constante de la conciencia natural. La conciencia natural sufre, en este ir más allá, un cambio que viene de la conciencia misma y que consiste en el imperio de la inquietud de la conciencia.

Se interpreta que Hegel quiere decir que la conciencia natural es lo todavía no verdadero, dominado por el cambio, la cual le arrebata hacia su verdad. De esta manera, la conciencia natural siente ese cambio como violencia y siente miedo por su propia existencia.

Nótese aquí la fuerza de la conciencia de sobreponerse a sí misma. La conciencia, a partir de la exposición, tiene como naturaleza su propia superación, idea que se puede retomar en otros ámbitos del pensamiento.

Dentro de una exposición lineal, tradicional, por llamarlo de alguna manera, no se consideraría el movimiento de la conciencia, como lo hacen los autores analizados, en donde se da un estado que podría ser placentero y único, y que podría tentar a la misma conciencia de quedarse en ese eterno presente de lo mismo y siempre igual, pero hay una superación que solo se puede realizar en el mundo del ser humano, de la conciencia, en este caso.

La filosofía es la primera que descubre la conciencia natural en su naturalidad y la reconoce. Bien es verdad que, en cambio, la filosofía hace caso omiso de la conciencia natural cuando ésta se jacta de ser filosofía para borrar los límites que la separan de la filosofía y volverle la espalda como conocer del ser de lo existente... (Heidegger, 1960: 138)

\section{Capítulo décimo}

Hegel (1960) retoma la temática de la conciencia refiriéndose a dos determinaciones "tal y como aparecen en la conciencia": las denomina saber y verdad. Estas determinaciones las llama "determinaciones abstractas", porque resultan de un mirar a la conciencia.

Heidegger reconstruye el razonamiento señalando el significado de conciencia (bewusstsein) en el sentido de que algo se halla en estado de sabido. Pero lo sabido está en el saber y es como un saber; es aquello a que se refiere la conciencia al modo del saber, es decir, lo sabido está en la relación y es, puesto que es para la conciencia, existe a la manera de "ser para...". Pero "ser para..." es un modo de saber, algo es para la conciencia, para lo que, como sabido, es aún otra cosa.

Ese ser en sí de lo sabido es lo que Hegel llama verdad. También la verdad es algo representado y un existente en sí para la conciencia. De esta manera, las dos determinaciones de la conciencia, saber y verdad, se distinguen entre sí, porque una es "ser para" y la otra es "ser en sí".

Asimismo, Hegel ha señalado un rasgo distinto de la conciencia con estas determinaciones. En la conciencia, hay algo que se distingue de ella y mediante ella; es algo así como si ella misma, mediante ella misma, fuera lo uno respecto de lo otro.

Lo distinguido en esta distancia (el objeto para el sujeto en el sujeto) sigue siendo referido empero por 
la distinción precisamente a lo que se pretende distinguir. La conciencia, al representar, se quita algo, pero se lo pone. La conciencia es en sí un distinguir que no lo es. La conciencia es ambigua en su esencia por ser esa distinción que no lo es. Ese ambiguo es la esencia del representar. (Heidegger, 1960: 141)

\section{Capítulo duodécimo}

Se caracteriza con una segunda proposición la naturaleza del objeto de exposición, que representa el saber aparente: "la conciencia se da la medida a ella misma" (Hegel citado por Heidegger, 1960: 142)

Heidegger se pregunta: ¿por qué dice Hegel "a ella misma" en lugar de "a sí misma"? Responde que lo hace, porque de la conciencia misma depende que haya una medida para ella; esta no se puede buscar en cualquier parte, sino que es ella misma.

"En ella misma" significa dos cosas: a) la conciencia tiene su medida en su esencia; b) lo que así se halla en ella, y no en cualquier otra cosa, no se lo da ya la conciencia precisamente a sí misma, se da la medida y no se la da, al mismo tiempo.

Significa que lo representado es lo verdadero, mientras la conciencia natural represente en sí lo existente y lo ciertamente "para ella", para esta conciencia que representa directamente:

Hegel emplea ese "para ella" -haciendo juego con el "para sí"- cuando quiere decir que la conciencia tiene por verdadero lo que ella representa francamente. Representando francamente, la conciencia se abre en lo representado y no se lo vuelve a referir a sí nuevamente, como lo que representa. Bien es verdad que la conciencia tiene su representado en su representar, pero no para sí, sino "para ella". (Heidegger, 1960: 143)

Además, con lo verdadero que la conciencia representa para ella, da al mismo tiempo el "para nosotros", que se fija en la verdad de lo verdadero. Esto ha dado en ella la verdad de lo verdadero, o sea, la medida: "El exponer nosotros el saber aparente como tal, tomamos al aparecer como criterio para medir por él el saber que ese aparente tiene por verdadero. En el saber aparente, su sabido es lo verdadero" (Heidegger, 1960: 143).

Heidegger estructura el pensamiento de Hegel negativamente, tratando de que parezca circular. Agrega que en lo que la conciencia tiene por su verdadero se realizará una forma de su verdad. Así, lo verdadero es el objeto "para ella"; la verdad es el objeto "para nosotros": "O sea, en lo que la conciencia declara que dentro de ella es lo 'verdadero' o 'en sí', tenemos nosotros (y precisamente nosotros lo que conocemos absolutamente) la medida que ella misma establece para medir con ella su saber" (Hegel citado por Heidegger, 1960: 145). De esta manera, en lo que aparece, el aparecer de lo que aparece, no aparece "para ella”, ni aparece para nosotros.

\section{Capítulo décimo tercero}

Hegel formula y aclara la tercera proposición sobre la conciencia: "la conciencia se examina a sí misma" (Hegel citado por Heidegger, 1960:145), lo cual es interpretado por Heidegger como si la conciencia es conciencia es el examinar. La palabra fundamental de la metafísica moderna -la conciencia- no se concibe hasta que en el "ser" se piensa a la vez el rasgo del examinar, y precisamente de un examinar que se determina mediante el ser-sabido del saber.

El hecho de que Hegel distinga todo eso, pero nivele las diferencias en un distinguir general y no las deje llegar a su derecho, tiene su fundamento oculto en la esencia de la metafísica, no en la posición metafísica fundamental de Hegel. De la esencia oculta de la metafísica proviene al mismo tiempo el hecho de que el nivel en que se nivelan las diferencias se determine por la discusión de lo uno y lo otro, discusión que se representa en la distinción de la ratio. "Hegel concibe la distinción como negación de la negación" (Heidegger, 1960: 147).

\section{Capítulo décimo cuarto}

El capítulo décimo cuarto expone la concepción de conciencia de Hegel como "experiencia", 
la cual se entiende como el ser de lo existente: "Este movimiento dialéctico que la conciencia ejerce en ella, tanto en su saber como en su objeto, puesto que para ella surge de ahí el nuevo objeto verdadero, es propiamente lo que se llama experiencia" (Hegel citado por Heidegger, 1960: 151). En este sentido, lo existente se ha convertido en sujeto y con este en objeto y en lo objetivo.

Se desprende, entonces, la nueva interpretación de Hegel acerca de que la experiencia ya no es el nombre de una clase de conocer, sino la palabra del ser, ya que este se percibe como tal desde lo existente.

Siguiendo la esencia de la conciencia: ser ya algo que al propio tiempo todavía no es, el ser en el todavía-no del ya, en consecuencia, ese ya está presente en el todavía no. El estar presente es en sí el referirse al ya y se pone de camino hacia el ya; se forma su camino. El ser de la conciencia hegeliana consiste en que se mueve.

Hegel denomina movimiento dialéctico a la experiencia. La exposición es propia de la esencia de la experiencia, esta es el aparecer de lo aparente como tal. Así la exposición de lo aparente pertenece al aparecer como al movimiento en que la conciencia realiza su realidad:

La conciencia es conciencia como diálogo entre el saber natural y el real, diálogo que consuma la reunión de su esencia mediante sus formas. En cuanto la formación de la conciencia se opera a la vez como diálogo que reúne y como reunión que se pronuncia, el movimiento de la conciencia es dialéctico. (Heidegger, 1960: 154)

Afirma Heidegger que la dialéctica no puede explicarse lógicamente a base de la posición y negación del representar, ni tampoco determinarse ónticamente como especial actividad y forma del movimiento dentro de la conciencia real.

Lo dialéctico, que es un modo de aparecer, es propio del ser que como realidad de lo existente se desarrolla a base del estar presente. Se afirma también que Hegel no concibe dialécticamente la experiencia; por lo contrario, piensa lo dialéctico a base de la esencia de la experiencia, es decir, la experiencia es la realidad de lo existente que se determina como subiectum a base de la subjetividad.

Dentro de la postura hegeliana, el objeto no se piensa como lo que esté en frente del representar, sino como aquello que surge como verdad de la conciencia: "La experiencia es el modo de la conciencia en cuanto es, sale en busca de su concepto que ella es en verdad" (Heidegger, 1960: 155)

La experiencia en su salida a buscar su concepto alcanza, en lo verdadero aparente, el aparecer de la verdad. De esta manera, llega al aparecer el aparecer mismo. Heidegger relaciona el caminar en el experimentar con su significado originario de salir: el experimentar es el llegar saliendo y alcanzando.

La experiencia es el modo de la presencia de lo presente, presencia que se presenta en el representarse. El mero objeto que surge a la conciencia, en cualquier momento en la historia de su formación, es la verdad de lo verdadero, es el ser de lo existente.

El último paso hacia la esencia de la experiencia como existencia de lo absoluto se dará en la última parte de la obra, tema que resumirá tanto la exposición hegeliana, vía Heidegger, como la demostración de la inversión de que la conciencia hace para ubicarse como subiectum en la imagen del mundo, punto final al que quiere arribar el autor.

\section{Capítulo décimo quinto}

Este capítulo, finalmente, se refiere a la representación que la conciencia natural tiene de lo que se denomina experiencia de Hegel.

Heidegger sale al acecho afirmando que dicha experiencia, pensada metafísicamente, permanece inaccesible a la conciencia natural, pues es la realidad de lo existente, y nunca se la podría encontrar como parte integrante existente dentro de lo existente. La experiencia se hace con otro objeto que se admite.

El objeto que se examina se ve desde las condiciones en que es traído por otros objetos en la experiencia ordinaria. Se pueden modificar las representaciones que hasta entonces se tenían 
del objeto. La no verdad del antiguo objeto se pone de manifiesto en el nuevo que se representa, a modo de relación comparativa con el objeto ya conocido, con el cual se quiere realizar la experiencia.

La conciencia natural en Hegel representa directamente su representado y su representar como existente. En consecuencia, debe lanzarse la conciencia natural para conservarse el ser de lo existente, según objetivo de Heidegger. De este modo, que vuelva a lo que para ella está en el estar-representado, en el representar de lo existente.

Por lo tanto, se pone de manifiesto que la conciencia ha abandonado ya el representar ordinario, con el aparecer de lo aparente, y ha pasado de lo aparente al aparecer, con lo cual se ha invertido: ${ }^{2}$

El rasgo fundamental de la experiencia de la conciencia es la inversión. Es aún "nuestro añadido". Lo que en esta inversión se expone a la conciencia no es "para la conciencia", es decir, no lo es para la natural. Lo que se expone en la inversión, no es "para ella", para la conciencia "que nosotros contemplamos", sino "para nosotros", los que contemplamos. (Heidegger, 1960: 157)

2. Véase cómo Heidegger quiere demostrar la inversión de la realidad, en cuanto interpreta que la conciencia natural toma como realidad su representar y, a partir de este, estructura su mundo. No puede ser de otra manera, el representar es la realidad. Si bien se puede traer a colación el principio "la realidad es mucho más rica que el concepto", aquí se habla del proceso de la conciencia. En este proceso, Hegel habla de la realidad: la conciencia. Es el movimiento de la conciencia en sí y para sí. Es la descripción del modo en que la conciencia se posesiona en la realidad. Heidegger tiene razón cuando apunta el olvido del ser, pero el posesionarse del ser lo da la conciencia, ¿cómo se comporta esta? Heidegger no quiere reconocerlo, por lo menos no da luz sobre un nuevo movimiento de la conciencia con el Ser de frente. Se tendría que volver al comportamiento y contemplación de la realidad que se da en el mundo griego, pero esta etapa ya fue analizada por Hegel, y coinciden, solo que Hegel la ve como un paso de la razón, un modo de ser, de la conciencia. Las condiciones objetivas y subjetivas de la existencia del ser humano no permiten volver a dicho estado de exaltación inicial; debe darse de otra manera, pero Heidegger no lo señala, tal vez, como se verá más adelante en las conclusiones.
Este "nosotros", prosigue Heidegger, es aquello que en la inversión de la conciencia natural, la dejan en su opinar, y que al mismo tiempo mira el aparecer de lo aparente, es el mirar en que se consuma su skepsis, que ha previsto la absolutez de lo absoluto y de antemano se ha sumido en ella.

Asimismo, la inversión de la conciencia que impera en la skepsis es el acto de precisión mediante el cual la conciencia se provee del aparecer mismo; en otras palabras, lo que se muestra es propio de la conciencia misma, es "para ella", es para la conciencia:

Pero el modo como lo aparente se muestra, o sea el aparecer es el aspecto de lo aparente, su eidos que forma todo lo aparente lo pone a la vista y lo configura: la forma. Hegel lo denomina lo "formal". (Heidegger, 1960: 158)

Así la exposición representa el ser de lo existente. La inversión es el añadido que se hace: es el hacerse patente de lo aparente como tal. Este añadido no impone a la experiencia algo que le sea extraño; más bien, se limita a sacar de ella misma lo que hay en ella como ser de la conciencia que es para sí misma su concepto. El añadido nunca supera el puro contemplar, necesario para la exposición; por lo contrario, en el añadido se inicia el contemplar puro.

Dentro de la interpretación de Heidegger, la experiencia es el ser de lo existente; mientras que lo existente aparece en el carácter de la conciencia y "es" como lo aparente en la representación.

Aparece ahora el concepto de la inversión, concepto e idea que será transcendental en el desarrollo de las ciencias sociales en general, y en la filosofía en particular. La inversión de la conciencia es la skepsis en la absolutez, invierte todo lo aparente en su aparecer, pues, de antemano, se provee del aparecer, lo abarca y abre el ámbito del lugar en que el aparecer se aparece.

La experiencia se anticipa a sí misma, en el volver se despliega en el estar presente de la conciencia y en estas condiciones pasa a ser constante, esta presencia constante la interpreta Heidegger como el ser de lo absoluto. 
De esta manera, la conciencia no se aparta de sí misma ni de su esencia; por lo contrario, esta enajenación es el sostenerse de la plenitud del aparecer con base en la voluntad, en donde domina la parousía (presencia o llegada) de lo absoluto.

Desde luego, plantea Heidegger, es muy diferente la cuestión de sí y hasta qué punto la subjetividad es destino esencial propio del ser en el que se "suprime" el desocultamiento del ser:

La inversión de la conciencia es la consumación del ver de la skepsis que ve, en la medida en que ya se ha provisto de la absolutez y en consecuencia está provisto de ella. El haber visto (vidi) de la skepsis es el saber de la absolutez. La inversión de la conciencia es el centro esencial del saber en que se despliega la exposición del saber aparente. De esta suerte, la exposición es la marcha de la conciencia misma al aparecerse en el aparecer. Es el "camino de la ciencia". (Heidegger, 1960: 161)

Entonces, dentro de la interpretación heideggeriana, la experiencia es el aparecer del saber aparente como aparente; la ciencia de la experiencia de la conciencia expone lo aparente como aparente, donde lo aparente es lo existente.

La skepsis de la exposición contempla lo aparente (en el aparecer) como lo presente y lo que en el aparecer ya predomina de sí mismo.

Resumiendo, entonces: la obra estudiada, en su totalidad, remite la conciencia natural a la apropiación de su morada, la cual se opera mediante la inversión de la conciencia y gracias a ella la conciencia llega a la experiencia en que sucede verdaderamente la parousía de lo absoluto.

La conciencia natural solo puede salir de su representar habitual y enderezarse a la experiencia si la remisión se realiza de tal modo que se enlace constantemente con las representaciones que la conciencia natural produce enseguida a su manera. Además, se produce de lo que le sale al paso con carácter de conocimiento absoluto; cuando se induce a la conciencia natural a disolver en ella misma el saber en que ella es, en cuanto ella es su concepto.

Este movimiento no es ni nada menos que la extrema enajenación de lo absoluto en su absolutez, según Heidegger. ¿Por qué enajenación? La respuesta está en que el hombre tiene que aceptar lo aparente tal como aparece.

Heidegger cita a Hegel para demostrar su hipótesis:

El saber que es nuestro objeto primero o directo, no puede ser otro que aquel que es saber directo, saber de lo "directo o existente". Por lo tanto, tenemos que comportarnos del mismo modo directo, o receptivo, es decir, no modificar nada en él de cómo se ofrece apartar, del concebir el comprender. (Hegel, citado por Heidegger, 1960: 173)

La exposición del aparecer de todo lo que tiene por existente y verdadero ha nacido de la certidumbre sensible, por lo que surge entonces su ser como objeto nuevo, la verdad de la certidumbre, o sea, la autoconciencia de que se sabe a sí misma.

\section{Conclusión}

Nuevamente, se observa una interpretación heideggeriana de la dialéctica hegeliana, donde se intenta detener un movimiento de la conciencia que va más allá de una cosificación de la realidad. Por lo contrario y por el momento, Hegel vitaliza la realidad en su movimiento, dotándola de una capacidad de ir más allá de ella misma siendo ella misma. Por lo tanto, sigue en su originalidad de ser en sí y para sí como referencia de la realidad. ${ }^{3}$

La manifestación de la realidad es la conciencia misma, aunque los conceptos análisis y síntesis no pertenecen a este mundo conceptual. Se puede ver cómo es uno en sí mismo, y cómo

3. "Si se separan concepto y realidad, el hombre ha muerto. Esta unión no tiene que ser solamente concebida como unidad en general, sino que la razón es esencialmente vitalidad, actividad; su actividad esencial consiste en que el concepto se produce, se convierte en contenido, pero de manera que lo producido está siempre de acuerdo con él. La realidad está siempre en dependencia de la idea, no existe por sí. Parece ser otro concepto, otro contenido, pero no es así. Lo que en la realidad es de otro modo que en el concepto, esta diferencia consiste sólo en la forma de la exterioridad. La realidad se hace idéntica al concepto" (Hegel, 1980: 49). 
se entrelazan en un solo movimiento de la razón, que ella misma es los dos momentos. ${ }^{4}$

$\mathrm{Si}$ se separan los distintos niveles, movimientos y modos de ser de la conciencia, es para efectos de visualizar la riqueza del movimiento y la capacidad de conciencia misma, no para quedarse en un eterno ir y venir del mismo punto. Es entonces un movimiento único, magistral, irrepetible en otros órdenes del desarrollo de la conciencia. ${ }^{5}$

La versión de la propuesta hegeliana, en interpretación personal nuestra, es una forma de afianzarse en la realidad, puesto que la verdad de lo aparente solo es verdad para la conciencia que la conoce. A no ser que veamos, hipotéticamente al estilo Einstein, un mundo paralelo donde existe otra conciencia al frente, que no necesariamente sería como la nuestra, y presente otro modo de ser de la realidad.

Lo más rico e interesante del planteamiento hegeliano, criticado por Heidegger porque lleva a una imagen del mundo, radica casualmente en que la propia conciencia es la comprometida consigo misma de crear y recrear, constantemente, la skepsis que le da un entendimiento, explicación y dominio sobre la realidad que es su realidad. No se trata de un solipsismo, sino de ubicar un espacio dentro de la razón donde se genera el

4. "Lo verdadero es el todo. Pero el todo es solamente la esencia que se completa mediante su desarrollo. De lo absoluto hay que decir que es esencialmente resultado, que solo al final es lo que es en verdad, y en ello precisamente estriba su naturaleza, que es la de ser real, sujeto o devenir de sí mismo. Aunque parezca contradictorio el afirmar que lo absoluto debe concebirse esencialmente como resultado, basta pararse a reflexionar un poco para descartar esta apariencia de contradicción” (Hegel, 1985: 16).

5. “(..) mientras que la antigua dialéctica sólo pretendía llevar a cabo, con la elaboración de las contradicciones, un trabajo preparatorio para el conocimiento, Hegel transforma esta tarea propedéutica o negativa de la dialéctica en una positiva (...) la fuerza del espíritu está en la síntesis como la mediación de todas las contradicciones (...) Hegel ve en la razón que concilia todas las contradicciones, la estructura universal de la realidad" (Gadamer, 1981: 132-133-134). conocimiento, el conocimiento de lo que se ha considerado la realidad.

Lo no real, para la conciencia, no existe, y esto mismo lo había aceptado ya Heidegger, cuando afirmó en su libro Introducción a la metafísica por qué el ser, y no más bien la nada. ${ }^{6}$

La versión heideggeriana sobre el concepto de conciencia en Hegel, más que una crítica a este, es un justificación de un temor de que dicha concepción, tal y como él la interpreta, justifique un uso e interpretación postmodernista, es decir, que el sujeto, creador de su mundo, se insensibilice con respecto a este y cree una imagen del mundo hecha a su medida, convirtiendo al ente, a la realidad, en una cosa disminuida en su valor; aún más, sin valor alguno en sí misma, sino que se relacione su ser a un proceso ajeno a la realidad misma.

En este sentido, la concepción hegeliana es más optimista que la concepción de Heidegger, en cuanto este visualiza un nihilismo, como catástrofe. Por lo contrario, Hegel ve un modo de ser de la conciencia en la búsqueda de la superación, una negación superadora, en cuanto en sí misma está su dialéctica superación.

Heidegger le da la espalda al aporte que hace Hegel sobre el comportamiento de la conciencia y su movimiento de experiencia y pone su esperanza, tal y como se expuso en el primer artículo, en un nuevo encuentro con el ser, mediado, en este caso, por el lenguaje.

Pero sabe que debe iniciarse el proceso de apertura del uno y el otro, del ser y el Dasein que será capaz de captar la manifestación de la

6. Claro está que la preocupación heideggeriana es ontológica, pero para efectos nuestros refleja la posición expuesta. “¿Por qué existe ser y no más bien, nada? He aquí el problema (...) es evidentemente la primera de todas las preguntas. Es la primera pregunta aunque no lo sea en relación con el orden y con la sucesión temporal de las preguntas" (Heidegger, 1982: 5). "Llega a un límite sólo en el ser no existente y en el ser jamás existente, en la nada. Todo lo que no signifique nada se comprende bajo esta pregunta y finalmente, abarca también la propia 'nada'; no porque sea algo, un ser, hablamos de ella, sino porque 'es' nada" (Heidegger, 1982: 6). 
realidad, pero ¿cuál es el camino del Dasein?, ¿cuál es el camino del ser en el mundo?, al estilo griego, que ya Hegel expuso y resumió en su momento.

Heidegger no ha resuelto el camino que tiene que recorrer la razón del Dasein, o bien, ¿quiere iniciar un nuevo camino sin tomar en cuenta lo recorrido hasta el momento? El recorrido anterior lo ha analizado y comentado y no ha extraído nada positivo.

Heidegger detiene el proceso dialéctico hegeliano y lo reduce a un modo de ser metafísico; disminuyéndolo ontológicamente, no se da cuenta de que él es parte del mismo movimiento.

Ser y pensar son lo mismo. Si el ser se manifiesta, es la razón quien lo capta dentro de un espacio y un tiempo, el espacio y el tiempo de la conciencia.

Finalmente, se considera que la teoría de la imagen del mundo y el olvido del ser no se han podido justificar con el sacrificio de la conciencia hegeliana. Dicha teoría de la imagen del mundo, ya era conocida en la época heideggeriana y sus contemporáneos la daban por un hecho. ${ }^{7}$

\section{Referencias bibliográficas}

Gadamer, Hans-Georg (1981). La dialéctica de Hegel. Cinco ensayos hermenéuticos (2da. Ed.; trad. de Manuel Garrido). Madrid: Cátedra.

Hegel, Georg Wilhelm Friedrich (1980). Introducción a la historia de la Filosofia (10ma. Ed.). Argentina: Aguilar.

Hegel, Georg Wilhelm Friedrich (1985). Prólogo a la Fenomenología del Espíritu (trad. Wenceslao Roces con la colaboración de Ricardo Guerra; 6ta. Reimpr.). México: Fondo de Cultura Económica.

Heidegger, Martín (1960). Sendas Perdidas (Holzwege; trad. de José Rovira Armengol). Buenos Aires: Losada.

HeIDEgGer, Martín (1982). Introducción a la metafísica (trad. de Hannelore Kruger). San José: Editorial Estudiantil, Federación de Estudiantes de la Universidad de la Universidad de Costa Rica.

Heisenberg, Werner (1962). Los nuevos fundamentos de la ciencia (trad. de la 9na. Ed. alemana por José Ma. Gimeno). Madrid: Norte-Sur.

7. "En la medida en que la acción práctica pasó a ocupar el núcleo de la imagen del mundo, los esquemas fundamentales del pensamiento perdieron su importancia absoluta. El espacio y el tiempo se convirtieron en objeto de la experiencia, perdiendo su contenido simbólico" (Heisenberg, 1962: 26). “...al menos en principio, una imagen del mundo, ha de poder abarcar todos los ámbitos de la realidad y en ella ha de quedar asignado un lugar determinado a cada uno de ellos" (Heisenberg, 1962: 52). "...separar lo subjetivo de lo objetivo lo considera el físico la primera condición previa de toda investigación" (Heisenberg, 1962: 69). "Mientras al principio era meta de toda investigación científica describir la naturaleza en lo posible como fuera en sí, es decir, sin nuestra intervención y si nuestra observación, hoy comprendemos que precisamente esta meta es inalcanzable" (Heisenberg, 1962: 82). 
